\title{
CONSUMO DE ALCOHOL EN ESTUDIANTES UNIVERSITARIOS COMO PREDICTOR PARA EL CONSUMO DE OTRAS DROGAS
}

\author{
ALCOHOL CONSUMPTION IN UNIVERSITY STUDENTS AS A PREDICTOR FOR THE CONSUMPTION OF OTHER \\ DRUGS
}

\author{
María del Carmen Gogeascoechea-Trejo, María Sobeida L. Blázquez-Morales, Patricia Pavón-León y María \\ Cristina Ortiz León
}

Universidad Veracruzana (México)

\begin{abstract}
Las diferentes encuestas muestran que el consumo de alcohol entre los jóvenes es alto, dicha edad coincide con el inicio de la vida universitaria. Asimismo, se considera que el uso de alcohol incrementa el riesgo de consumir drogas ilegales. Objetivo. Conocer la prevalencia de consumo de alcohol en los estudiantes universitarios y su asociación como predictor del consumo de drogas ilegales. Método. Estudio transversal analítico en 19,815 estudiantes, mediante el análisis de la base de datos del Segundo Diagnóstico de Percepción, Riesgo y Consumo de Drogas en Estudiantes Universitarios en una universidad pública del estado de Veracruz, México. Resultados. La prevalencia del consumo de alcohol fue de 55.5\%. El análisis de regresión logística mostró que los estudiantes que consumieron alcohol registraron mayor probabilidad de consumir drogas ilegales con respecto a los que no reportaron consumo de alcohol. Conclusiones. Se confirma la asociación entre el consumo de alcohol con drogas ilegales, datos que podrían reafirmar al alcohol como droga que es puerta de entrada para el consumo de otras. Es una tarea primordial para las universidades llevar a cabo acciones de intervención para la promoción de estilos de vida saludables, prevención y detección oportuna del consumo de alcohol y otras drogas.
\end{abstract}

Palabras clave: consumo de alcohol, drogas ilícitas, estudiantes, universidad.

Different surveys show that alcohol consumption among young people is high, this age coincides with the beginning of university life. Likewise, it is considered that the use of alcohol increases the risk of consuming illegal drugs. Objective. To determine the prevalence of alcohol consumption in university students and its association as a predictor of illegal drug consumption. Method. Cross-sectional study in 19815 students, through the analysis of the database of the Second Diagnosis of Perception, Risk and Drug Use in University Students carried out at a public university in the state of Veracruz, Mexico. Results. The prevalence of alcohol consumption was $55.5 \%$. Logistic regression analysis showed that students who consumed alcohol had a higher probability of consuming illegal drugs compared to those who did not report alcohol consumption. Conclusion. The association between alcohol consumption and illegal drugs is confirmed, data that could reaffirm alcohol as a drug that is a gateway for the consumption of others. It is a primary task for universities to carry out intervention actions for the promotion of healthy lifestyles, prevention and timely detection of the consumption of alcohol and other drugs.

Keywords: alcohol consumption, illicit drugs, students, university.

María del Carmen Gogeascoechea-Trejo, Instituto de Ciencias de la Salud, Universidad Veracruzana, México, https://orcid.org/00000002-2646-4079

María Sobeida L. Blázquez-Morales, Instituto de Ciencias de la Salud, Universidad Veracruzana, México, https://orcid.org/00000003-3763-9337

Patricia Pavón-León, Instituto de Ciencias de la Salud, Universidad Veracruzana, México, https://orcid.org/0000-0002-1868-453

María Cristina Ortiz León, Instituto de Salud Pública, Universidad Veracruzana, México, correspondencia a cortiz@uv.mx, http://orcid.org/0000-0003-4953-5076. 
El consumo de alcohol se ha reconocido como una práctica socialmente aceptada, principalmente en los jóvenes. El Informe sobre la situación mundial del alcohol y la salud 2018, señala que 3 millones de personas murieron (5.3\% de todas las muertes) en el mundo durante 2016 debido al consumo nocivo del alcohol. Asimismo, menciona que la mortalidad resultante del consumo de alcohol es mayor que la causada por enfermedades como la tuberculosis, la infección por el $\mathrm{VIH} /$ sida y la diabetes. En todo el mundo, el alcohol fue la causa del $7.2 \%$ de la mortalidad prematura (en las personas de 69 años y menores). Las personas más jóvenes se vieron desproporcionadamente afectadas por el alcohol en comparación con las personas mayores, y $13.5 \%$ de las muertes de quienes se encuentran entre los 20 y los 39 años se atribuyen al alcohol, principalmente debidas a traumatismos, enfermedades digestivas, enfermedades cardiovasculares, así como cáncer (Organización Panamericana de la Salud, 2019a).

El Informe también menciona que en el mundo la prevalencia de los episodios de consumo excesivo de alcohol alcanza su punto máximo a la edad de 20 a 24 años. Los jóvenes de 15 a 24 años, cuando son bebedores, a menudo beben en sesiones de consumo excesivo, por lo tanto, las tasas de prevalencia de los episodios de consumo excesivo de alcohol en los bebedores de 15 a 24 años son más altas que en el resto de la población. La prevalencia de los episodios de este tipo de consumo es particularmente elevada en los hombres (Organización Panamericana de la Salud, 2019a).

En la región de las Américas, en el año 2016, el alcohol ocasionó 379,000 muertes (5.4\% de todas las muertes). Al igual que la tendencia mundial, en dicha región en el mismo año, $47 \%$ de todos los hombres mayores de 15 años consumidores actuales de alcohol y $20.3 \%$ de todas las mujeres, informaron haber tenido consumo excesivo episódico de alcohol en el último mes. Asimismo, el consumo promedio de todos los bebedores en conjunto fue de casi tres bebidas diarias todos los días del año. (Organización Panamericana de la Salud, 2019b).

En México, las diferentes encuestas nacionales sobre el tema reportan que la tendencia del consumo de alcohol va en aumento; la Encuesta Nacional de Consumo de Drogas, Alcohol y Tabaco 2016-2017 (ENCODAT) reporta que en la población de 18 a 65 años en las mediciones de 2011 y 2016, la prevalencia en el último año aumentó de 53.3\% a 55.7\%, la prevalencia de consumo de alcohol en el último mes registró un aumento de 35\% a 39.9\%. Asimismo, por patrón de consumo, el consumo excesivo en el último año y en el último mes mostró un aumento entre 2011 y 2016, tanto en hombres como en mujeres. El promedio de la edad de inicio de bebidas alcohólicas fue de 17.9 años en 2016, por sexo los hombres reportaron iniciar el consumo de alcohol a los 16.7 años y las mujeres a los 19.2 años. Las encuestas anteriores muestran a los jóvenes como una población vulnerable que coincide con el inicio de la vida universitaria, etapa en la cual el consumo de alcohol puede derivar en trastornos a nivel mental que redundan en problemas académicos como un bajo rendimiento escolar, depresión, así como problemas familiares. Igualmente, el uso de alcohol incrementa el riesgo de consumir drogas ilegales, así como la práctica de conductas sexuales de riesgo.

Los estudios realizados sobre el uso y abuso de alcohol en estudiantes universitarios muestran que la prevalencia de consumo de alcohol entre los universitarios es alta, al igual que el consumo de alto riesgo o de dependencia, siendo los hombres los mayores consumidores, quienes presentan una frecuencia de consumo de una vez a la semana, predominando un patrón de fin de semana. Asimismo, la mayoría de los estudiantes reconocen haberse embriagado alguna vez en su vida con afectaciones a nivel académico. (Armendariz, Villar, Alonso, Alonso \& Oliva, 
2014; Betancourth-Zambrano, Tacán-Bastidas \& Cordoba-Paz, 2017; Castaño-Pérez \& CalderónVallejo, 2014; Davoren, Shiely, Byrne \& Perry, 2015; Fernández-Castillo et al., 2016; Gómez, Landeros, Noa \& Patricio, 2017; Latorres \& Huidobro, 2012; Sepúlveda, Roa \& Muñoz, 2011)

Los estudios sobre consumo de alcohol como predictor del consumo de otras drogas, son escasos, algunos de ellos se han llevado a cabo en Estados Unidos, Argentina y México en población entre 11 y 24 años, los cuales han mostrado que los usuarios de alcohol presentan mayor probabilidad de iniciar el uso de drogas ilegales. (Herrera-Vázquez, Wagner, VelascoMondragón, Borges \& Lazcano-Ponce, 2004; Morello, Pérez, Peña, Braun, Thrasher \& Mejía, 2019; Griffin, Lowe, Botvin, \& Acevedo, 2019)

Considerando que el consumo de alcohol es un predictor para el consumo de otras drogas, es necesario que las Instituciones de Educación Superior, cuenten con un registro sistematizado que aporte conocimiento adecuado de la situación de sus estudiantes, que sirva para el desarrollo de intervenciones que permitan reducir el consumo de alcohol y de esta manera prevenir el uso de otras drogas. Por tal motivo, en la Universidad Veracruzana, la Red Veracruzana de Investigación en Adicciones (REVIVA) realizó en el año 2017, el Segundo Diagnóstico de Percepción, Riesgo y Consumo de Drogas en Estudiantes Universitarios. A partir de este Segundo Diagnóstico, se planteó como objetivo del presente proyecto conocer la prevalencia de consumo de alcohol en los estudiantes universitarios y su asociación como predictor del consumo de drogas ilegales.

\section{Método}

\section{Diseño y participantes}

Se trata de un estudio cuantitativo transversal analítico. La información procede de la base de datos del Segundo Diagnóstico de Percepción, Riesgo y Consumo de Drogas en Estudiantes Universitarios realizado en 2017 en una universidad pública del estado de Veracruz, México. Se incluyeron los datos de 19815 estudiantes de todos los semestres de todos los programas educativos en todas las áreas académicas: económico-administrativa, ciencias de la salud, técnica, humanidades, biológico-agropecuaria y artes, que de manera voluntaria aceptaron participar en el estudio.

\section{Instrumento}

Para la recolección de la información se utilizó el cuestionario denominado Consumo de Drogas en Estudiantes Universitarios (CODEU-2), que consta de 273 preguntas distribuidas en seis apartados, de los cuales para fines del presente estudio, se seleccionaron las siguientes variables: a) características sociodemográficas (edad, sexo, estado civil, donde vive); b) características académicas (área académica, semestres que cursan considerando iniciales con uno a tres semestres cursados, intermedios de cuatro a seis, avanzados de siete a nueve y rezagados más de 10 semestres); c) consumo de alcohol en los últimos doce meses; d) características del consumo de alcohol (edad de inicio, frecuencia, número de copas); y e) consumo de drogas ilegales (fármacos de uso médico (estimulantes/tranquilizantes) sin prescripción, marihuana, cocaína, crack, alucinógenos, inhalables, metanfetaminas, heroína y otras sustancias las cuales se catalogaron como drogas emergentes) en los últimos doce meses. El instrumento fue validado utilizando el Alpha de Cronbach, obteniendo un índice de confiabilidad de .787 lo que permite establecer que se trata de un instrumento confiable. 


\section{Procedimiento}

El instrumento se aplicó en línea y fue publicado en la plataforma LimeSurvey habilitada por el Centro de Estudios de Opinión y Análisis de la propia universidad. Se invitó a participar a los estudiantes de todas las áreas académicas, facultades y semestres a través de una campaña denominada Percíbete2, cuya promoción se realizó en todos los medios de difusión de la universidad. El proyecto contó con la aceptación y el apoyo de las autoridades universitarias, lo que significa que se obtuvieron los apoyos necesarios para promocionar el proyecto entre los estudiantes e invitarlos a responder el cuestionario en línea desde sus propios dispositivos o desde los centros de cómputo de la misma universidad. Asimismo, el proyecto fue revisado y aprobado por el Comité de Ética en Investigación del Instituto de Ciencias de la Salud de la Universidad Veracruzana con folio 003/2015.

Al inicio de la encuesta en línea, se les informó a los estudiantes que su participación era voluntaria y que la información proporcionada sería con fines de investigación, además se les garantizó el anonimato y la confidencialidad ya que no se les solicitó el nombre o número de matrícula.

\section{Análisis de datos}

Se realizó un análisis estadístico descriptivo de las variables mediante frecuencias y porcentajes, para analizar las diferencias entre consumidor y no consumidor de alcohol se utilizó la prueba estadística $\chi 2$. Se consideraron con significancia estadística cuando $p<.05$. Como medida de asociación se realizaron análisis de regresión logística binaria simple para estimar las probabilidades mediante Odds Ratio (OR) con intervalos de confianza (IC) de 95\%, bajo el nivel de significancia de 0.05 . Se consideró como variable independiente el consumo de alcohol en los últimos doce meses y como variables dependientes el consumo de cada droga ilegal en los últimos doce meses. Para el procesamiento de la información se utilizó el programa SPSS versión 23 para Windows.

\section{Resultados}

Del total de los estudiantes que participaron en el estudio, 10995 reportaron consumo de alcohol en los últimos doce meses, por lo tanto, la prevalencia de consumo fue de 55.5\%.

En la tabla 1 se observa que, de los estudiantes que consumían alcohol, 58.7\% eran mujeres y $41.3 \%$ hombres, mientras que de los estudiantes que no consumían alcohol, la mayoría eran mujeres (65.1\%), diferencias estadísticamente significativas $(p=.001)$. La mayoría de los estudiantes de los diferentes grupos de edad consumían alcohol, el grupo de mayor consumo fue el de 17 a 32 años (67.1\%) y el de menor consumo los estudiantes que presentaban edad mayor o igual a 26 años con un porcentaje de $6.1 \%$ ( $p=.001)$.

Con respecto al estado civil, tanto los solteros como los casados reportaron consumo de alcohol en los últimos doce meses, la mayoría eran solteros (94.1\%) ( $p=.049)$. (Tabla 1 ).

En lo que se refiere al área académica a la que pertenecían, la mayoría de los estudiantes de todas las áreas reportaron consumo de alcohol ( $p=.001)$, siendo las de mayor porcentaje el área Económico-administrativa con $33.1 \%$ y Ciencias de la salud con 31.6\%. En cuanto a los semestres, sin importar el semestre que cursaban, la mayoría de los estudiantes consumían alcohol, el 
porcentaje más alto de consumo se presentó en los que estudiantes que cursaban los semestres intermedios (45.9\%) ( $p=.001)$. (Tabla 1$)$.

Tabla 1

Distribución de las variables académicas y sociodemográficas de los estudiantes según consumo de alcohol en los últimos doce meses

\begin{tabular}{|c|c|c|c|c|c|c|c|}
\hline & \multicolumn{7}{|c|}{ Consumo de alcohol en los últimos doce meses } \\
\hline & \multicolumn{2}{|c|}{$\mathrm{Si}$} & \multicolumn{2}{|c|}{ No } & \multicolumn{2}{|c|}{ Total } & \multirow[b]{2}{*}{$p$} \\
\hline & $f$ & $\%$ & $f$ & $\%$ & $f$ & $\%$ & \\
\hline \multicolumn{8}{|l|}{ Sexo } \\
\hline Hombre & 4546 & 41.3 & 3079 & 34.9 & 7625 & 38.5 & \multirow{2}{*}{.001} \\
\hline Mujer & 6449 & 58.7 & 5741 & 65.1 & 12190 & 61.5 & \\
\hline \multicolumn{8}{|l|}{ Edad } \\
\hline 17 a 21 años & 7376 & 67.1 & 6518 & 73.9 & 13894 & 70.1 & \multirow{3}{*}{.001} \\
\hline 22 a 25 años & 2946 & 26.8 & 1919 & 21.8 & 4865 & 24.6 & \\
\hline$\geq 26$ años & 673 & 6.1 & 383 & 4.3 & 1056 & 5.3 & \\
\hline \multicolumn{8}{|l|}{ Estado civil } \\
\hline Soltero & 10343 & 94.1 & 8317 & 94.3 & 18660 & 94.2 & \multirow{2}{*}{.049} \\
\hline Casado/unión libre & 652 & 5.9 & 503 & 5.7 & 1155 & 5.8 & \\
\hline \multicolumn{8}{|l|}{ Área académica } \\
\hline Económico-administrativa & 3644 & 33.1 & 3171 & 36.0 & 6815 & 34.4 & \multirow{6}{*}{.001} \\
\hline Ciencias de la salud & 3475 & 31.6 & 2608 & 29.6 & 6083 & 30.7 & \\
\hline Técnica & 2048 & 18.6 & 1630 & 18.5 & 3678 & 18.6 & \\
\hline Humanidades & 1075 & 9.8 & 928 & 10.5 & 2003 & 10.1 & \\
\hline Biológico-agropecuaria & 602 & 5.5 & 442 & 5.0 & 1044 & 5.3 & \\
\hline Artes & 151 & 1.4 & 41 & 0.5 & 192 & 1.0 & \\
\hline \multicolumn{8}{|l|}{ Semestres cursados } \\
\hline Iniciales & 3611 & 32.8 & 3362 & 38.1 & 6973 & 35.2 & \multirow{4}{*}{.001} \\
\hline Intermedios & 5042 & 45.9 & 3981 & 45.1 & 9023 & 45.5 & \\
\hline Avanzados & 1916 & 17.4 & 1262 & 14.3 & 3178 & 16.0 & \\
\hline Rezagados & 426 & 3.9 & 215 & 2.4 & 641 & 3.2 & \\
\hline
\end{tabular}

Fuente: Base de datos del Segundo Diagnóstico de Percepción, Riesgo y Consumo de Drogas en Estudiantes Universitarios, 2017.

Los estudiantes que mencionaron consumir bebidas alcohólicas en los últimos doce meses, $56.8 \%$ iniciaron el consumo de esta substancia entre los 11 y los 17 años; 36\% una frecuencia de consumo de una vez al mes o menos, $67.7 \%$ un consumo de cuatro copas o menos por ocasión. Asimismo $82 \%$ mencionaron que nunca les había sucedido que no pudieran dejar de beber una vez que habían empezado. (Tabla 2).

Tabla 2 
Edad de inicio y características del consumo de alcohol en el último año por los estudiantes

\begin{tabular}{lcc}
\hline & $f$ & $\%$ \\
\hline Edad de inicio & 114 & 1.0 \\
$\leq 10$ años & 6243 & 56.8 \\
11 a 17 años & 4638 & 42.2 \\
$\geq 18$ años & & \\
Frecuencia de consumo & 3270 & 29.8 \\
Una a tres veces al año & 3962 & 36.0 \\
Una vez al mes o menos & 2894 & 26.3 \\
Dos a cuatro veces al mes & 763 & 6.9 \\
Dos a tres veces por semana & 106 & 1.0 \\
Cuatro o más veces por semana & & \\
Número de copas & 7443 & 67.7 \\
Cuatro o menos & 3552 & 32.3 \\
Cinco o más & & \\
Frecuencia no pudo dejar de beber una vez que había & & 82.0 \\
empezado & 9012 & 12.0 \\
Nunca & 1315 & 3.7 \\
Menos de una vez al mes & 406 & 1.9 \\
Mensualmente & 209 & 0.4 \\
Semanalmente & 53 & \\
Diario o casi a diario & & \\
\hline
\end{tabular}

Fuente: Base de datos del Segundo Diagnóstico de Percepción, Riesgo y Consumo de Drogas en Estudiantes Universitarios, 2017.

En cuanto al consumo de drogas ilegales, $0.6 \%$ de los estudiantes con consumo de alcohol en los últimos doce meses reportaron consumo de estimulantes $y / 0$ tranquilizantes sin prescripción médica, de los que no consumían alcohol $0.1 \%$ reportó haber consumido dichas sustancias, diferencias estadísticamente significativas ( $p=.001)$.

Referente al consumo de marihuana, $15.9 \%$ y $1.0 \%$ de los estudiantes con y sin consumo de alcohol respectivamente, mencionaron haber consumido dicha sustancia $(p=.001)$.

El consumo de cocaína lo reportaron $1.3 \%$ de los estudiantes con consumo de alcohol y nadie de los que no consumieron lo notificó. La misma tendencia se presentó en el consumo de otras drogas ilegales en los estudiantes que consumieron alcohol con respecto a los que no consumieron, con diferencias estadísticamente significativas ( $p=.001$ ) (Tabla 3$)$.

Tabla 3

Consumo de alcohol y consumo de drogas ilegales en los últimos doce meses

\section{Consumo de alcohol en los últimos doce meses}

\begin{tabular}{|c|c|c|c|c|c|}
\hline \multicolumn{2}{|c|}{ Si } & \multicolumn{2}{|c|}{ No } & \multicolumn{2}{|c|}{ Total } \\
\hline$f$ & $\%$ & $f$ & $\%$ & $f$ & $\%$ \\
\hline
\end{tabular}

Consumo de estimulantes y/o tranquilizantes sin prescripción 


\begin{tabular}{|c|c|c|c|c|c|c|c|}
\hline $\mathrm{Si}$ & 118 & 1.1 & 9 & 0.1 & 127 & 0.6 & \multirow{2}{*}{.001} \\
\hline No & 10877 & 98.9 & 8811 & 99.9 & 19688 & 99.4 & \\
\hline \multicolumn{8}{|c|}{ Consumo de marihuana } \\
\hline $\mathrm{Si}$ & 1745 & 15.9 & 84 & 1.0 & 1829 & 90.8 & \multirow{2}{*}{.001} \\
\hline No & 9250 & 84.1 & 8736 & 99.0 & 17986 & 9.2 & \\
\hline \multicolumn{8}{|c|}{ Consumo de cocaína } \\
\hline $\mathrm{Si}$ & 144 & 1.3 & 2 & 0.0 & 146 & 0.7 & \multirow{2}{*}{.001} \\
\hline No & 10851 & 98.7 & 8818 & 100.0 & 19669 & 99.3 & \\
\hline \multicolumn{8}{|c|}{ Consumo de crack } \\
\hline $\mathrm{Si}$ & 32 & 0.3 & 3 & 0.0 & 35 & 0.2 & \multirow{2}{*}{.001} \\
\hline No & 10963 & 99.7 & 8817 & 100.0 & 19780 & 99.8 & \\
\hline \multicolumn{8}{|c|}{ Consumo de alucinógenos } \\
\hline $\mathrm{Si}$ & 220 & 2.0 & 11 & 0.1 & 231 & 1.2 & \multirow{2}{*}{.001} \\
\hline No & 10775 & 98.0 & 8809 & 99.9 & 19584 & 98.8 & \\
\hline \multicolumn{8}{|c|}{ Consumo de inhalables } \\
\hline Si & 27 & 0.2 & 2 & 0.0 & 29 & 0.1 & \multirow{2}{*}{.001} \\
\hline No & 10968 & 99.8 & 8818 & 100.0 & 19786 & 99.9 & \\
\hline \multicolumn{8}{|c|}{ Consumo de metanfetaminas } \\
\hline $\mathrm{Si}$ & 94 & 0.9 & 0 & 0.0 & 94 & 0.5 & \multirow{2}{*}{.001} \\
\hline No & 10901 & 99.1 & 8820 & 100.0 & 19721 & 99.5 & \\
\hline \multicolumn{8}{|c|}{ Consumo de heroína } \\
\hline $\mathrm{Si}$ & 8 & 0.1 & 1 & 0.0 & 9 & 0.0 & \multirow{2}{*}{.041} \\
\hline No & 10987 & 99.9 & 8819 & 100.0 & 19806 & 100 & \\
\hline \multicolumn{8}{|c|}{ Consumo de otros opiáceos } \\
\hline $\mathrm{Si}$ & 27 & 0.2 & 3 & 0.0 & 30 & 0.2 & \multirow{2}{*}{.001} \\
\hline No & 10968 & 99.8 & 8817 & 100.0 & 19785 & 99.8 & \\
\hline \multicolumn{8}{|c|}{ Consumo de otras sustancias } \\
\hline $\mathrm{Si}$ & 46 & 0.4 & 7 & 0.1 & 53 & 0.3 & \multirow{2}{*}{.001} \\
\hline No & 10949 & 99.6 & 8813 & 99.9 & 19762 & 99.7 & \\
\hline
\end{tabular}

Fuente: Base de datos del Segundo Diagnóstico de Percepción, Riesgo y Consumo de Drogas en Estudiantes Universitarios, 2017.

En el análisis de asociación, en la Tabla 4 se observa que en los estudiantes que consumieron alcohol en los últimos doce meses existe una mayor probabilidad (OR=10621, IC95\% 5.38920.930) de consumir estimulantes sin prescripción médica con respecto a los que no reportaron este consumo. Referente al consumo de tranquilizantes sin prescripción médica la probabilidad fue de 6.177, IC95\% 4.216-9.048; para el consumo de marihuana es de 19.619 IC95\% 15.73124.469; para el consumo de cocaína 58.510 IC95\% 14.490-236.272; para el consumo de crack 8.579 IC95\% 2.626-28.024; para el consumo de alucinógenos 16.351 IC95\% 8.918-29.978; para el consumo de inhalables 10.854 IC95\% 2.580-45.654; para el consumo de opiáceos 7.235 IC95\% 2.194-23.856; para el consumo de otras sustancias no reportadas anteriormente 5.289 IC95\% 2.387-11.721. Todos estos resultados fueron estadísticamente significativos. 
Tabla 4

Regresión logística binaria simple para identificar si el consumo de alcohol en los últimos doce meses se asocia como predictor de consumo de drogas ilegales

\begin{tabular}{|c|c|c|c|c|c|c|c|}
\hline & B & $\mathrm{EE}$ & Wald & gl & $p$ & OR & IC95\% \\
\hline Consumo de estimulantes & 2.363 & .346 & 46.605 & 1 & .000 & 10.621 & {$[5.389,20.930]$} \\
\hline $\begin{array}{ll}\text { Consumo } & d \epsilon \\
\text { tranqulizantes } & \end{array}$ & $e_{1.821}$ & .195 & 87.370 & 1 & .000 & 6.177 & {$[4.216,9.048]$} \\
\hline Consumo de mariguana & 2.977 & .113 & 697.591 & 1 & .000 & 19.619 & {$[15.731,24.469]$} \\
\hline Consumo de cocaína & 4.069 & .712 & 32.650 & 1 & .000 & 58.510 & {$[14.490,236.272]$} \\
\hline Consumo de crack & 2.149 & .604 & 12.663 & 1 & .000 & 8.579 & {$[2.626,28.024]$} \\
\hline Consumo de alucinógenos & s 2.794 & .309 & 81.622 & 1 & .000 & 16.351 & {$[8.918,29.978]$} \\
\hline Consumo de inhalables & 2.385 & .733 & 10.583 & 1 & .001 & 10.854 & {$[2.580,45.654]$} \\
\hline Consumo de opiáceos & 1.979 & .609 & 10.568 & 1 & .001 & 7.235 & {$[2.194,23.856]$} \\
\hline $\begin{array}{l}\text { Consumo de otra } \\
\text { sustancias }\end{array}$ & 1.666 & .406 & 16.836 & 1 & .000 & 5.289 & {$[2.387,11.721]$} \\
\hline
\end{tabular}

Nota: $\mathrm{EE}=$ Error estándar; $\mathrm{gl}$ = Grados de libertad; Sig= significancia; OR=Odds Ratio; IC=Intervalo de confianza.

\section{Discusión}

Los datos en la población universitaria estudiada demuestran que más de la mitad (55.5\%) de los estudiantes consumen alcohol, cifra menor a la encontrada en investigaciones previas en estudiantes universitarios por Armendariz et al., 2014; Betancourth-Zambrano et al., 2017; Castaño-Pérez \& Calderón-Vallejo, 2014; Davoren et al., 2015; Fernández-Castillo et al., 2016; Gómez et al., 2017; Latorres \& Huidobro, 2012; Sepúlveda et al., 2011; cuyas cifras oscilan entre $64.2 \%$ y $97.5 \%$. Sin embargo, la prevalencia en el presente estudio fue mayor a la reportada por Mantilla-Toloza, Villamizar \& Peltzer, 2016; Saltos. 2011. Asimismo, la prevalencia de consumo de alcohol en el último año registrada en la ENCODAT para la población de 18 a 65 años fue de $55.7 \%$, similar a la del presente estudio.

Se observó una relación entre el sexo y el consumo de alcohol, con mayor prevalencia en las mujeres, dichos resultados coinciden sólo con un estudio (Davoren y et al., 2015) y se contradicen con la mayoría de los estudios en estudiantes universitarios donde se ha reportado una mayor prevalencia en los hombres (Armendariz-García, Villar, Alonso, Alonso \& Oliva, 2012; Betancourth-Zambrano et al., 2017; Gómez et al., 2017; Mantilla-Toloza et al., 2016; Montaño. Morales, Gómez, Vera \& Gantiva, 2011; Nieto, 2011; Restrepo, Agudelo, Giraldo \& Sánchez, 2011; Salcedo, Palacios \& Espinosa, 2011; Saltos. 2011). No obstante, aun cuando las mujeres se encuentran más representadas en la muestra, este estudio corrobora un incremento significativo en el consumo en las mujeres, lo cual es preocupante dada la mayor susceptibilidad de las mujeres a los efectos nocivos del alcohol.

El grupo de edad en el que predomina el consumo de alcohol es el de 17 a 21 años, disminuyendo en los siguientes grupos de edad, dicha tendencia coincide con los semestres de estudio, ya que los resultados muestran que en los semestres intermedios existe un mayor porcentaje de consumo, y a medida que avanzan los años de estudios universitarios, el porcentaje 
de consumo disminuye, algunos autores denominan a este fenómeno como la adaptabilidad al estrés que presentan los estudiantes de los semestres avanzados y a un mayor conocimiento de los riesgos y los efectos nocivos del consumo de alcohol (Latorres \& Huidobro, 2012; MantillaToloza et al., 2016; Méndez \& Azaustre, 2017;).

La edad de inicio del consumo de alcohol en la mayoría de los estudiantes fue en la adolescencia, es decir en menores de 17 años, etapa en la que aún no han ingresado a la universidad, dichos resultados coinciden con la mayoría de los estudios al respecto, lo cual evidencia una tendencia al consumo en edades cada vez más tempranas, destacando la influencia que pudieran tener los factores de riesgo psicosociales específicamente en esta etapa la denominada "presión de pares" (Castaño-Pérez \& Calderón-Vallejo, 2014; Castaño, García \& Marzo, 2014; Fernández-Castillo et al., 2016; Nieto, 2011; Salcedo et al., 2011; Sepúlveda et al., 2011; Saltos. 2011).

Con respecto a la frecuencia del consumo de alcohol se observa que predomina el consumo una vez al mes, quincenal y entre una a tres veces al año, tal y como lo reportan diversos estudios (Betancourth-Zambrano et al., 2017; Davoren et al., 2015; Lema, Varela, Duarte \& Bonilla, 2011; Salcedo et al., 2011), sin embargo, hay estudiantes que mencionan un consumo mayor al semanal, lo que implica un riesgo para la dependencia. A lo anterior, se suma que $32 \%$ de los estudiantes reportan un consumo de cinco copas o más por ocasión y $6 \%$ mencionan una frecuencia mensual o menor, de no haber podido dejar de beber una vez que habían iniciado el consumo.

El consumo de drogas ilegales constituye un problema que afecta a los jóvenes. De los estudiantes encuestados, $16.9 \%$ reportaron consumo de alguna droga ilegal y de éstos, la mayoría habían consumido alcohol en los últimos doce meses, lo que corrobora el policonsumo en los estudiantes universitarios reportado en diversos estudios (Betancourth-Zambrano et al., 2017; Davoren et al., 2015; Sepúlveda et al., 2011; Saltos. 2011). La droga ilegal más consumida fue la marihuana, seguida por el consumo de estimulantes o tranquilizantes, cocaína y alucinógenos, datos que coinciden con otros estudios (Gómez et al., 2017; Sepúlveda et al., 2011; Saltos. 2011).

En el presente estudio se presentó como principal hallazgo que en los consumidores de alcohol aumenta la probabilidad de consumo de drogas ilegales. Los resultados confirman la asociación entre el consumo de alcohol como predictor para el consumo de drogas ilegales, datos que podrían reafirmar la teoría respecto al alcohol como droga que es puerta de entrada para el consumo de otras. Dichos resultados son consistentes con otras investigaciones, mismas que reportan que el consumo de alcohol incrementa el riesgo para el consumo de otras drogas (Davoren et al., 2015; Gómez et al., 2017; Saltos, 2011; Sepúlveda et al., 2011), sin embargo, ninguna de estas investigaciones ha estimado la probabilidad para dicho consumo.

El consumo de alcohol es un fenómeno que se encuentra presente en los estudiantes universitarios, debido a la independencia y autonomía que presentan una vez que inician la vida universitaria, convirtiéndose en un problema de salud pública, tanto en términos de riesgo para algunos trastornos físicos y mentales como para el inicio en el consumo de otras drogas, dando como resultado problemas académicos, familiares e incluso legales.

Para las Instituciones de Educación Superior, es una tarea primordial garantizar la formación de mejores profesionales para lo cual es fundamental llevar a cabo acciones que aborden la promoción de estilos de vida saludables a través de la formación integral de sus estudiantes, la 
prevención del consumo mediante la inclusión de temáticas relacionadas en los planes de estudio y en su caso, educar respecto del consumo responsable de bebidas alcohólicas. Asimismo, es necesario desarrollar estrategias de intervención para la detección del abuso del alcohol y consumo de drogas ilegales, con el fin de canalizar a los estudiantes a instancias que abordan esta problemática.

Entre las limitaciones del estudio se encuentra que estos resultados no se pueden generalizar a toda la población debido a que se trata de una muestra no probabilística y sólo aplican para esta población universitaria, sin embargo, muestran una problemática que se encuentra presente en el contexto universitario y que es necesario evidenciar con el fin de que sean tomados en cuenta en las políticas públicas orientadas al consumo de drogas lícitas e ilícitas.

\section{Conflicto de interés}

Los autores declaran que no existen conflictos de interés.

\section{Financiación}

La presente investigación no fue financiada por ningún organismo ni institución.

\section{Referencias}

Armendáriz, G. N. A., Villar, L. M .A., Alonso, C. M. M., Alonso, C. B. A., \& Oliva, R. N. N. (2012). Eventos estresantes y su relación con el consumo de alcohol en estudiantes universitarios. Investigación en Enfermería: Imagen y Desarrollo, 14(2), 97-112.

Armendáriz, G. N. A., Alonso, C. M. M., Alonso, C. B. A., López, C. M. A., Rodríguez, P. L. A., \& Méndez, R. M. D. (2014). La familia y el consumo de alcohol en estudiantes universitarios. Ciencia y enfermería $X X, \quad 3, \quad 109-118$. http://dx.doi.org/10.4067/s0717$\underline{95532014000300010}$

Betancourth-Zambrano, S., Tacán-Bastidas, L., \& Cordoba-Paz, E. G. (2017). Consumo de alcohol en estudiantes universitarios colombianos. Rev Univ. Salud, 19(1), 37-50. doi: http://dx.doi.org/10.22267/rus.171901.67

Castaño-Pérez, G. A., \& Calderón-Vallejo, G. A. (2014). Problemas asociados al consumo de alcohol en estudiantes universitarios. Rev. Latino-Am. Enfermagem, 22(5), 739-46. doi: $\underline{10.1590 / 0104-1169.3579 .2475}$

Castaño P. G. A., García, C. J. A., \& Marzo, C. J. C. (2014). Consumo de alcohol y factores intervinientes en estudiantes universitarios. Revista Cubana de Salud Pública, 40(1), 47-54.

Davoren, M. P., Shiely, F., Byrne, M., \& Perry, I. J. (2015). Hazardous alcohol consumption among university students in Ireland: a cross-sectional study. BMJ Open 5: e006045. doi:10.1136/ bmjopen-2014-006045

Fernández-Castillo, E., Molerio, P. O., Fabelo, R. J. R., Sánchez, H. D., Cruz, P.A., \& Grau, A. R. (2016). Consumo de tabaco y alcohol en estudiantes universitarios cubanos. Revista del Hospital Psiquiátrico de La Habana, 13(2). 
Gómez, C. Z., Landeros, R. P., Noa, P. M., \& Patricio, M. S. (2017) Consumo de alcohol, tabaco y otras drogas en jóvenes universitarios. Revista de Salud Pública y Nutrición, 16(4), 1-9.

Griffin, K., Lowe, S., Botvin, C., \& Acevedo, B. (2019). Patterns of adolescent tobacco and alcohol use as predictors of illicit and prescription drug abuse in minority young adults. J Prev Interv Community, 47(3), 228-242. doi: 10.1080/10852352.2019.1603672

Herrera-Vázquez, M., Wagner, F. A., Velasco-Mondragón, E., Borges, G., Lazcano-Ponce, E. (2004). Inicio en el consumo de alcohol y tabaco y transición a otras drogas en estudiantes de Morelos, México. Salud Publica Mex, 46, 132-140.

Latorres, M., \& Huidobro, A. (2012). Prevalencia de consumo de alcohol en estudiantes de la Facultad de Medicina en la Universidad Católica del Maule. Rev Med Chile, 140, 1140-1144.

Lema, L. F., Varela, M. T, Duarte, C., \& Bonilla, M. V. (2011). Influencia familiar y social en el consumo de alcohol en jóvenes universitarios. Rev. Fac. Nac. Salud Pública, 29(3), 264-271

Lorenzo, M., Cajaleón, B., \& Gutiérrez, E. (2012). Prevalencia y factores asociados al consumo de alcohol y tabaco en estudiantes de nutrición de una universidad de Lima-Perú. Revista Peruana de Epidemiología, 16(3), 01-05.

Mantilla-Toloza, S. C., Villamizar, C. E., \& Peltzer, K. (2016). Consumo de alcohol, tabaquismo y características sociodemográficas en estudiantes universitarios. Rev Univ. Salud, 18(1), 715.

Méndez, G. J. M., \& Azaustre, L. M. C. (2017). El consumo de alcohol en universitarios. Estudio de las relaciones entre las causas y los efectos negativos. Rev. complut. educ. 28(3), 689-704. doi: http://dx.doi.org/10.5209/rev RCED.2017.v28.n3.49725

Montaño, M. R., Morales, G. A., Gómez, M. M. A, Vera, M. A., \& Gantiva D. C. A. (2011). Consumo de alcohol en estudiantes universitarios. Psicogente, 14(25), 27-35.

Morello, P., Pérez, A., Peña, L., Braun, S. N., Thrasher, J. F. Mejía, R. (2019). Factores predictores del consumo de sustancias en adolescentes argentinos: estudio longitudinal. Rev Argent Salud Pública, 10(40), 32-38.

Nieto, E. I. (2016). Consumo de alcohol entre los estudiantes de la Universidad de Extremadura. Metas Enferm, 19(8), 6-11.

Organización Panamericana de la Salud. (2019a). Informe sobre la situación mundial del alcohol y la salud 2018. Resumen. Washington, D.C.: OPS.

Organización Panamericana de la Salud. (2019b). Sinopsis de política. Políticas tributarias y de precios del alcohol en la Región de las Américas. Washington, D.C.: OPS.

Restrepo, M. L., Agudelo M. A., Giraldo T. M. T., \& Sánchez, D. L. (2011). Factores de contexto asociados al consumo de alcohol en estudiantes universitarios. Revista CES Salud Pública, 2(2), 136-147. 
Salcedo, M. A., Palacios, E. X., \& Espinosa, A. F. (2011). Consumo de alcohol en jóvenes universitarios. Avances en Psicología Latinoamericana, 29(1), 77-97.

Saltos, S. M. (2011). Estudio del consumo de drogas en estudiantes universitarios. Revista Ciencia UNEMI, 4(5), 8-17.

Sepúlveda, M. J., Roa, J., \& Muñoz, M. (2011). Estudio cuantitativo del consumo de drogas y factores sociodemográficos asociados en estudiantes de una universidad tradicional chilena. Rev Med Chile, 139, 856-863. 